\title{
Simplified numerical method for understanding the aeroelastic response of line slender structures under vortex shedding action
}

\author{
A. Vasallo ${ }^{1}$, A. Lorenzana ${ }^{1}$, A. Foces ${ }^{1}$ \& C. E. Lavín ${ }^{2}$ \\ ${ }^{1}$ Department of Architectural Constructions, Ground Engineering \\ and Mechanics of Continuous Means and Theory of Structures, \\ Valladolid University, Spain \\ ${ }^{2}$ Structural Design Area, CARTIF, Spain
}

\begin{abstract}
The lock-in and drag amplification phenomenon is studied in a flexible cantilever using a simplified fluid-structure interaction (FSI) algorithm. NavierStokes equations for incompressible flow have been solved in $2 \mathrm{D}$ in several transverse planes of the line-like structure. A fractional step scheme has been used to solve the fluid field. In each fluid plane, the displacements have been taken into account considering an Arbitrary Lagrangian Eulerian (ALE) approach. The stabilization of convection and diffusion terms has been achieved by means of orthogonal quasi-static subscales. No turbulence model has been included. In order to solve the structural problem, a monodimensional element for thin walled cross-section beams has been considered. The standard second order Newmark method has been used to include the dynamical behaviour equations. The wind has been considered to be an incompressible fluid acting on the structure in a series of planes that are independent among them, and transverse to the structure. For each period of time, the fluid problem was solved, the aeroelastic analysis was carried out, and the geometry of the mesh of each fluid plane was updated according to the structure displacements.
\end{abstract}

Keywords: vortex-induced vibrations, line slender structures, aeroelasticity, coupled problems, lock-in, drag amplification. 


\section{Introduction}

Vibrations generated by vortex shedding may occur in slender structures and are very important for the design of these kinds of structures, such as chimneys, towers, masts, cables or bridge-decks. The practical significance of vortexinduced vibrations (VIV) has led to a considerable amount of research work, either experimental or theoretical, the latter through nonlinear-oscillator modelling approaches and, recently, also through Computational Fluid Dynamics (CFD). In the comprehensive review of Williamson and Govardhan [1], fundamental results and discoveries concerning VIV are discussed.

The first numeric researches on air flow around a cylinder were carried out in 1969 by Son and Hanratty [2]. In 1986 Braza et al. [3] used the finite volume method to analyse the vortex shedding in cylinders, with a Reynolds number less than 1000. In 1993, Dawes [4] used the finite volume method to solve the same problem. He used an adaptive method, including the ability to refine the mesh in function of the solution obtained, to get a computationally economical solution. Kalktsis et al. [5] used the spectral element method to carry out a computational research of the forces on a cylinder harmonically oscillating in a perpendicular direction to the uniform flow. Other authors, such as Jan and Sheu [6] and Prasanth et al. [7,8], have also used the finite element method to analyse the vortex shedding phenomenon.

Other researchers, such as Rodi [9] in 1997, studied the vortex shedding phenomenon in non aerodynamic bodies. Steggel and Rockliff [10] used the discrete vortex method. With this same method, Larsen and Walther [11] were able to calculate aerodynamic parameters, such as the lift and drag coefficients or the Strouhal number, for different cross-sections. The numerical methodology to analyse the lock-in phenomenon, and its relation with the use conditions of slender structures to limit their vibration levels, was used by Lopes et al. [12]. For analysing the air flow and its action on the structure, these researchers used a method based on the finite volumes technique, while the dynamic behaviour of the structure was calculated with the finite element method (FEM).

In this work vortex-induced vibrations of line slender structures have been studied using a simplified fluid-structure interaction algorithm. An example of the response of a cantilever beam subjected to the wind action is shown in a range where vibration behaviour is critical, resulting in lock-in and drag amplification effects.

\section{Lock-in and drag amplification phenomenon}

Vortex-induced vibrations occur when vortices are shed alternatively from opposite sides of the structure whose primary frequency $n_{s}$ is, according to the Strouhal relation,

$$
n_{s}=\frac{S_{t} D}{U}
$$

where $S_{t}$ depends upon body geometry and the Reynolds number, $D$ is the across-wind dimension of the body, and $U$ is the mean velocity of the uniform 
flow in which the body is immersed. This gives rise to a fluctuating force density $F_{y}(t)$ perpendicular to the wind direction, which can be rendered dimensionless and expressed in terms of lift coefficient as

$$
C_{L}=\frac{F_{y}(t)}{\frac{1}{2} \rho U^{2} D}
$$

where $\rho$ is the fluid density.

Under the action of the vortices shed in its wake the structure will be driven periodically, but this driving will lead only small response unless the vortex shedding frequency approaches the natural across-flow natural frequency of the structure $n_{e}$. Near this frequency greater body movement is elicited, and the structural motion begins to interact strongly with the flow in such a way that the vortex shedding frequency synchronizes with the structure's natural frequency which controls the vortex-shedding phenomenon even when variations in flow velocity modify the nominal Strouhal frequency away from the natural frequency by a few percent. This phenomenon is called lock-in and it is observed for low values of mass and/or structural damping [13].

On the other hand, an interesting large-amplitude response mode is found at higher reduced velocities outside the principal synchronization range [14,15], resulting in a large amplification of the drag coefficient. This vibration mode is due to a coupled streamwise-transverse motion, where the streamwise amplitude becomes non-negligible. There is strong correlation between the streamwise and transverse motions, as the frequency of the transverse oscillation is exactly half the frequency for the streamwise vibrations.

\section{Theoretical formulation}

To study the lock-in and drag amplification phenomenon a one-dimensional structural model [16] has been coupled to the fluid flow. In order to perform the interaction between both problems, it is necessary to identify the geometry of the across-section of the beam and the distribution of pressure or force density on its boundary. Under the usual assumptions, the motion of the beam axis describes the motion of the whole cross-section, which can be imagined as a rigid surface which follows the translation and the rotation of the beam axis. This allows one to associate any section of finite dimensions lying in the $x y$ plane to each point of the one-dimensional beam element, assumed to be oriented as the $O z$ axis. Given the hypothesis of small-strains, the motion of the cross-section outside of the $x y$ plane can be neglected. This is not consistent with the kinematics hypothesis on the beam motion, but can be accepted as a very good approximation for the only purpose of describing the motion of the beam imagined as a three dimensional body.

Current work concentrates on the case of blunt-body cross-sections with a clear predominance of the shape resistance over the friction resistance. For this category of structures, the dynamic behaviour of the overall coupled system 
plays a very important role. A time-accurate viscous flow solver is thus needed. Even if the features of the problem make it possible to use a rather coarse mesh, it can be immediately verified that the number of elements needed for a complete 3D simulation makes it unviable for any realistic beam length.

In our case, the interest focuses on the behaviour of a beam subjected to a flow orthogonal to the beam axis. Under this assumption, the fluid domain was modelled with a number of independent two-dimensional planes of fluid where, on each one, the problem can be solved separately. Conceptually, the solution on each fluid plane provides a force density $F_{y}(t)$, acting on the cross-section beam, which is obtained by integrating the pressure of the fluid over the corresponding cross-section boundary. A time-varying distributed load over the beam is obtained by linear interpolation between consecutive fluid planes. The deformation of the beam provides a relation between the motion of the different sections.

It is interesting to make a conceptual consideration on the nature of the coupling. The beam formulation makes use of the small strain hypothesis, which implies that the reference and deformed configuration are considered to coalesce in writing the equilibrium. On the other hand, the motion of the cross-section is obtained exactly once given the motion of the corresponding axis, without taking advantage of the small strain hypothesis. This implies that the loads acting on the structure will be allowed depending in a non-linear way on the motion of the beam. This feature may become important for the cases in which the model moves at the limits of the small strain formulation.

Finally, it is needed to choose a suitable coupling algorithm. In this work, the aeroelastic problem is characterized by large Reynolds numbers and flows featuring sharp separations at the corners of the section. It is well known that loose coupling procedures are very efficient for such a problem. A fractional-step approach was chosen and is described in Rossi [17]. It assumes the form:

- Solve the structure using the loads (pressure) at time.

- Move the fluid-mesh according to the structural motion.

- Solve the fluid.

- Solve the structural problem applying the newly calculated pressure. In detail, the structural solution assumes the form

- Integrate the fluid force on the beam section.

- Solve the dynamic problem.

- Move the section accordingly to the calculated motion of the beam.

- Assign a velocity to the points of the section consistently with the mesh motion scheme chosen.

The fluid solver used is a fractional-step scheme based on a second order pressure splitting [18]. The solver allows an Arbitrary Lagrangian Eulerian (ALE) description of the fluid domain, allowing dealing simply with the deformation of the fluid domain. The stabilization of convection and diffusion terms is obtained using the Orthogonal Sub Scales (OSS) approach [19]. It is assumed that the temporal variation of the subscales is negligible. No turbulence model is included in the simulation. The motion equations of the structure are integrated using a standard second-order accurate Newmark scheme. 


\section{Steel mast subjected to wind action}

The lock-in and drag amplification phenomenon is studied in a $34 \mathrm{~m}$ steel mast under wind action. The diameter of the mast is $0.5 \mathrm{~m}$ and the shell thickness is $4.8 \mathrm{~mm}$. The elastic properties are Young's modulus $E=2.1 \cdot 10^{11} \mathrm{~Pa}$, shear modulus $G=8.077 \cdot 10^{10} \mathrm{~Pa}$, Poisson's ratio $v=0.3$ and density $\rho=7772 \mathrm{~kg} / \mathrm{m}^{3}$. The mast was modelled as a flexible cantilever with four beam elements and structural damping, proportional to the mass matrix, is assumed. The first natural frequency is estimated by a modal analysis to be $0.43 \mathrm{~Hz}$.

The fluid is air with density $\rho=1.21 \mathrm{~kg} / \mathrm{m}^{3}$ and dynamic viscosity $\mu=1.8^{\circ} \cdot 10^{-}$ ${ }^{5} \mathrm{Ns} / \mathrm{m}^{2}$. Wind action was modelled as unique plane of fluid with triangular finite elements located at the free end of the cantilever (see fig. 1).
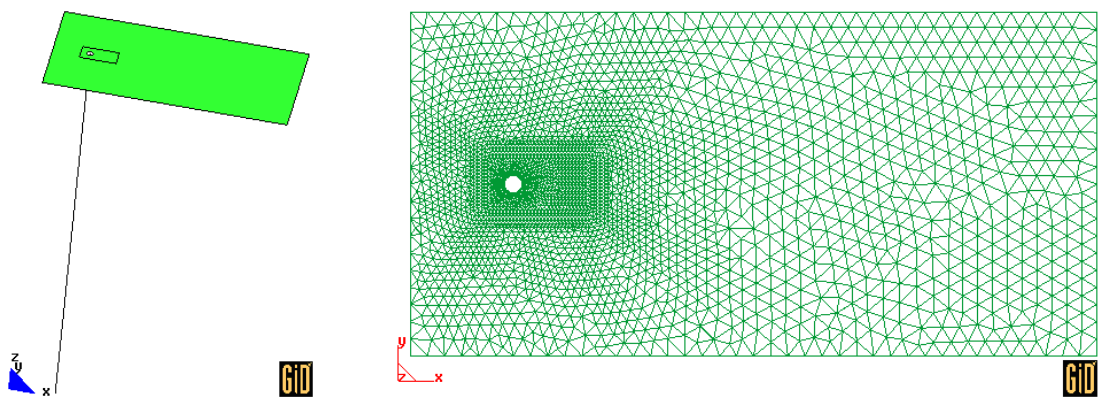

Figure 1: Mast model under wind action and fluid domain mesh.

Figure 2 shows the frequency response of the across-wind vibration of the structure vs. reduced velocity for different structural damping ratios $\beta_{s}$. The variation of the transverse amplitude response of the cantilever with the reduced velocity on a normal scale and logarithm scale is plotted in fig. 3. It is observed that in the principal synchronization regime (typically $U_{r}$ about 5) the lock-in phenomenon occurs only for the case in which the structural damping ratio is 0.025 and results in high response amplitude.

A further interesting result for the cantilever is the large-amplitude transverse oscillation for high velocities. It is noticed that the smaller the structural damping is, the more quickly the large-amplitude response occurs, that is, the lower fluid flow velocity is required. In order to explain this, we consider the case of structural damping ratio $\beta_{s}=0.025$ and $U_{r}=9$ as representative of the rest.

Figure 4 shows the oscillation of the lift coefficient, eqn. (2), with time and the FFT analysis. We can see the synchronization between the vortex-shedding frequency and the structure's natural frequency. During the first seconds of the analysis $(t<150 \mathrm{sec}$. approximately), the transverse displacements are small and the frequency of the lift coefficient is $0.84 \mathrm{~Hz}$. This value is due to the vortex shedding and is coherent with the Strouhal relation, eqn. (1), for $S_{t}=0.21(\operatorname{Re}=$ $\left.6.67 \cdot 10^{4}\right)$. For a time $t$ between 150 and $250 \mathrm{sec}$. approximately, the synchronization occurs between the two frequencies. Finally, for a time $t>250$ 


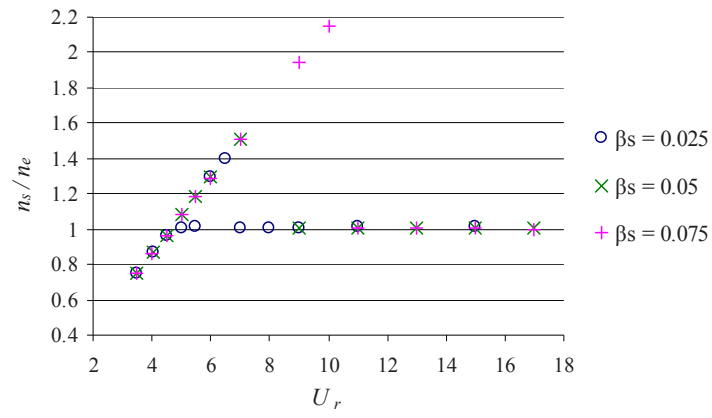

Figure 2: $\quad$ Frequency response vs. reduced velocity.

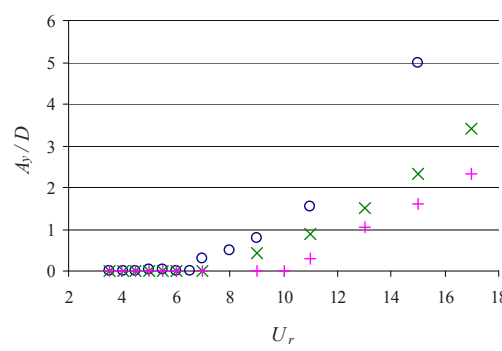

$\circ \beta \mathrm{s}=0.025 \times \beta \mathrm{s}=0.05+\beta \mathrm{s}=0.075$

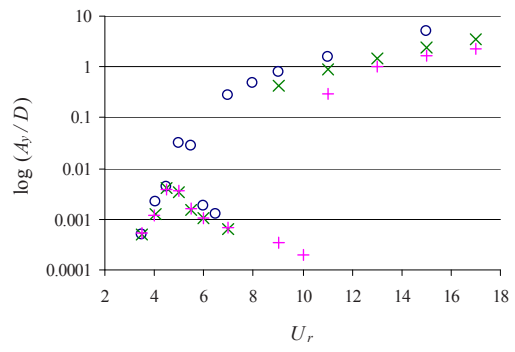

$\circ \beta \mathrm{s}=0.025 \times \beta \mathrm{s}=0.05+\beta \mathrm{s}=0.075$

Figure 3: Transverse amplitude response vs. reduced velocity.

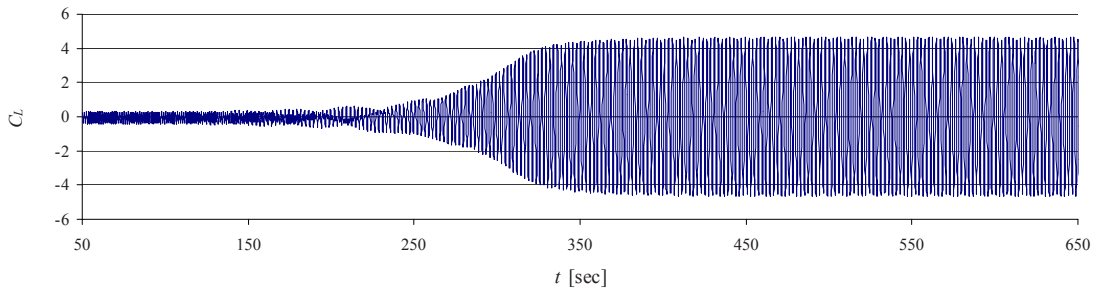

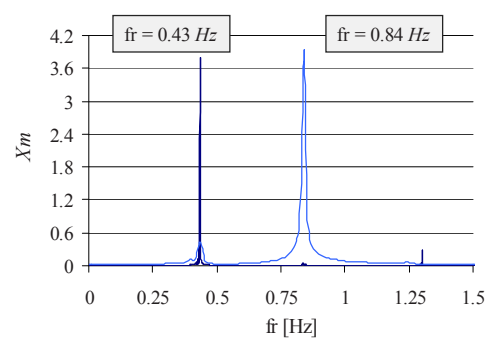

$-\mathrm{t}>250 \mathrm{sec}$

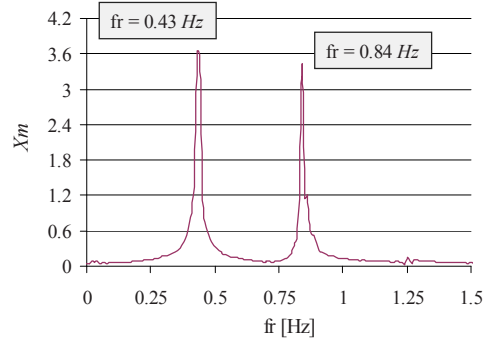

$-150<\mathrm{t}<250 \mathrm{sec}$

Figure 4: $\quad$ Oscillation of the lift coefficient and FFT analysis. 


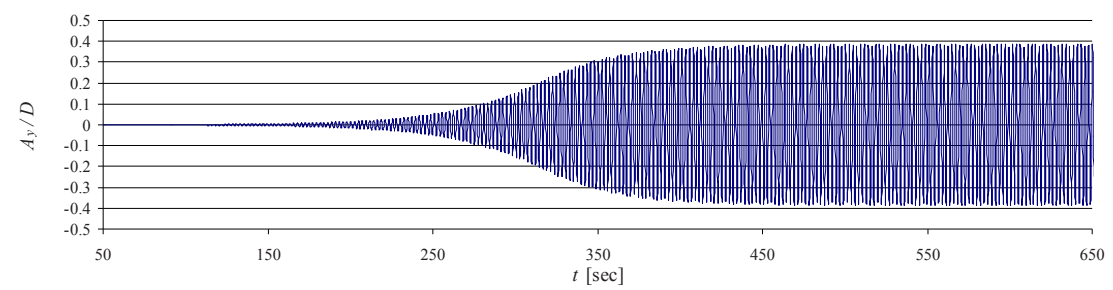

Figure 5: Time history of the across-wind vibration.

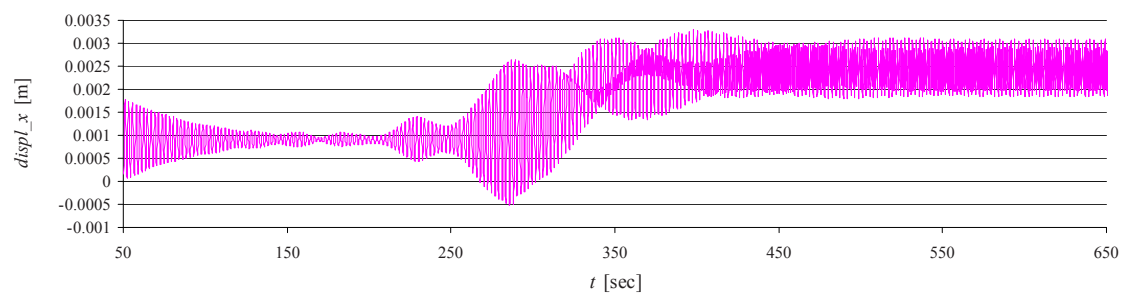

Figure 6: Time history of the across-wind and the along-wind vibration.

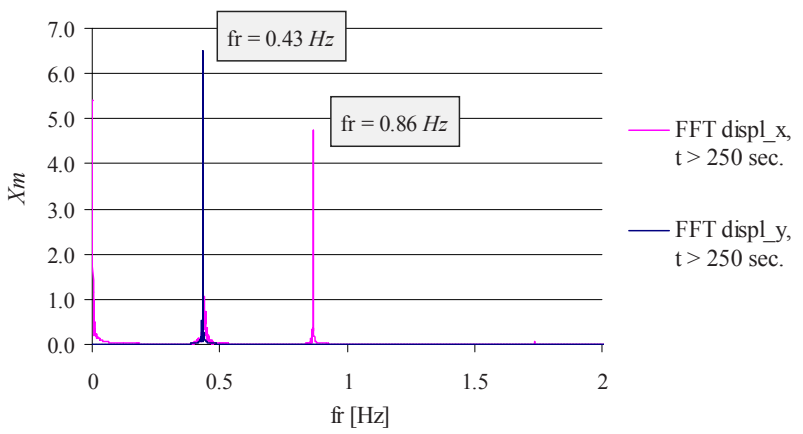

Figure 7: Frequency response of the across-wind and the along-wind vibration.

sec. approximately, the frequency of the fluctuating lift coefficient is equal the natural frequency $(0.43 \mathrm{~Hz})$ and the transverse displacements increase significantly (see fig. 5) due to structural motion begins to interact strongly with the wind field and the structure's natural frequency controls the vortex-shedding phenomenon.

Time history of the along-wind vibration is showed in fig. 6. We can observe that the structure vibration increases and occurs around a new position of equilibrium for a time $t>250 \mathrm{sec}$. approximately. For this time, there is a strong correlation between streamwise and transverse oscillations, as we find that the frequency of the across-wind vibration is $0.43 \mathrm{~Hz}$ and the frequency of the along-wind direction is twice as much as transverse one, $0.86 \mathrm{~Hz}$ (see fig. 7). 


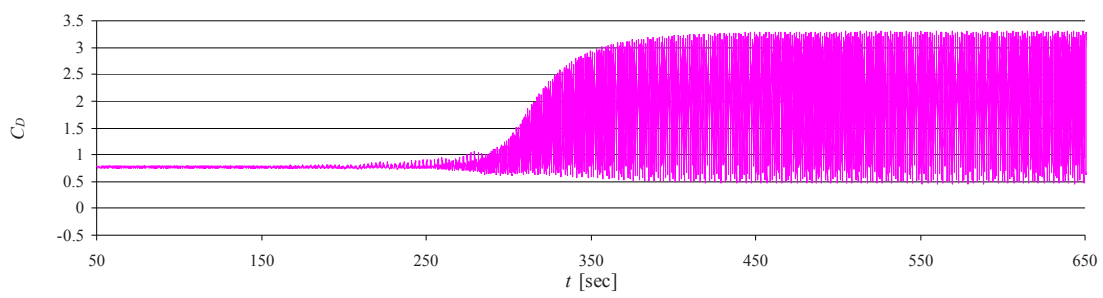

Figure 8: $\quad$ Oscillation of the drag coefficient.

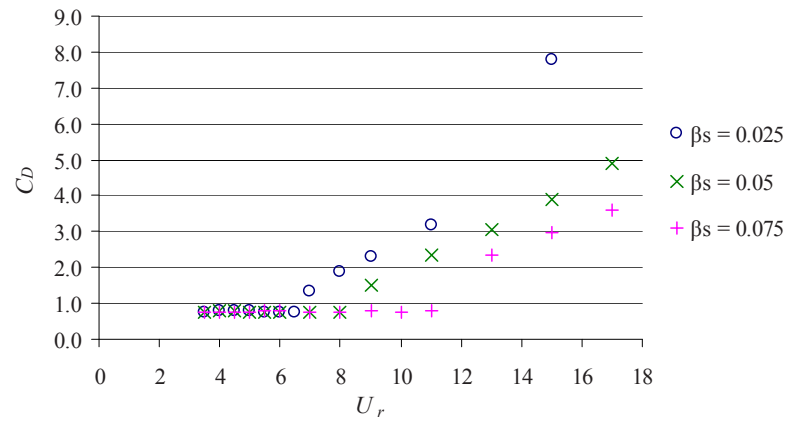

Figure 9: $\quad$ RMS value of the drag coefficient vs. reduced velocity.

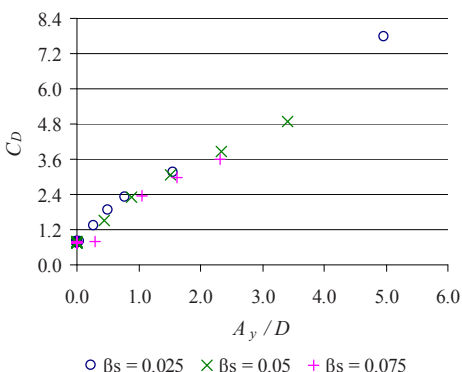

$\circ \beta \mathrm{s}=0.025 \times \beta \mathrm{s}=0.05+\beta \mathrm{s}=0.075$

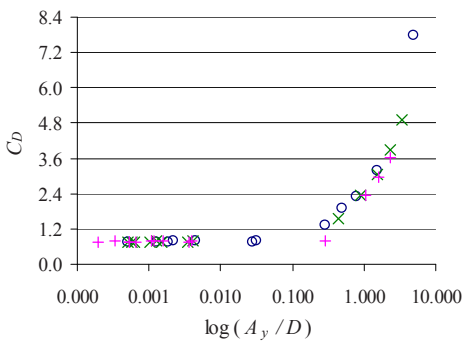

$\circ \beta \mathrm{s}=0.025 \times \beta \mathrm{s}=0.05+\beta \mathrm{s}=0.075$

Figure 10: $\quad$ RMS value of the drag coefficient vs. transverse displacement.

The variation of the drag coefficient with time is showed in fig. 8. It is noticed that the drag coefficient increases significantly (drag amplification) as a result of the coupling between streamwise and transverse motion that happens for a time $t>250 \mathrm{sec}$. approximately.

Figure 9 shows the RMS value of the drag coefficient vs. reduced velocity for different structural damping ratios. It is observed that the smaller the structural damping is, the more quickly the RMS value of the drag coefficient increases from a certain value of the velocity. The variation of the RMS value of the drag coefficient with the transverse displacement is showed in fig. 10 on a normal and logarithm scale. We can see that the increase of the drag coefficient depends on the amplitude of the transverse vibration of the structure but it is independent of the structural damping ratio. 


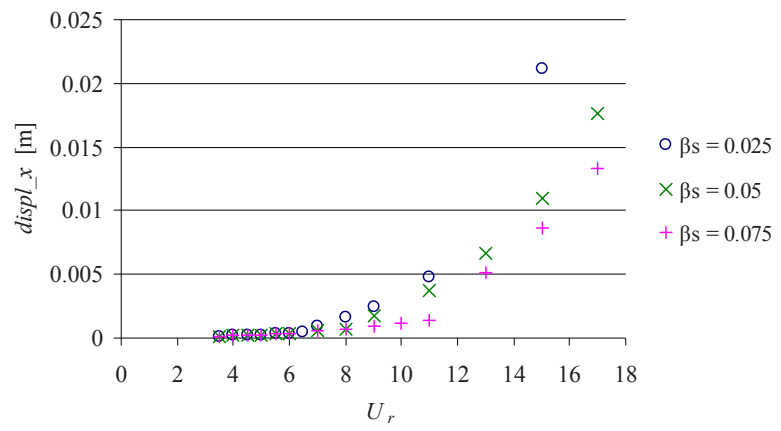

Figure 11: $\quad$ RMS value of the along-wind displacement vs. reduced velocity.

Finally, Figure 11 shows the increase of the along-wind displacement of the structure as the reduced velocity increases. This variation of the along-wind displacement is in line with the variation of the drag coefficient.

\section{Conclusions}

In summary, a simplified numerical method for fluid-structure interaction (FSI) analysis of line slender structures subjected to the action of the wind has been developed. The lock-in and drag amplification phenomenon has been studied successfully in a $34 \mathrm{~m}$ steel mast. A high-speed vibration mode of largeamplitude response has been found, which is outside the principal synchronization range, and is associated with a streamwise-transverse vibration coupling. The frequency of the transverse vibration of this high-speed mode corresponds to half the streamwise vibration frequency, also according to experimental results.

\section{References}

[1] Williamson, C.H.K., \& Govardhan, R., A brief review of recent results in vortex induced vibrations, Journal of Wind Engineering and Industrial Aerodynamics, 96(6-7), pp. 713-735, 2008.

[2] Son J.S., \& Hanratty, T.J., Numerical Solution for the Flow around a Cylinder at Reynolds Number of 40, 200, 500, Journal of Fluid Mechanics, 35, pp. 369-386, 1969.

[3] Braza, M., Chassaing, P., \& Minh, H.H., Numerical Study and Physical Analysis of the Pressure and Velocity fields in the near Wake of a Circular Cylinder, Journal of Fluid Mechanics, 165, pp. 79-130, 1986.

[4] Dawes, W.N., Simulating unsteady turbomachinery flows on unstructured meshes which adapt both in time and space, International Gas Turbine and Aeroengine Congress and Exposition, Cincinnati, Ohio, 1993.

[5] Kalktsis L, Triantafyllou, G.S., \& Ozbas, M., Excitation, inertia, and drag forces on a cylinder vibrating transversely to a steady flow, Journal of Fluids and Structures, 23(1), pp. 1-21, 2007. 
[6] Jan, Y.J. \& Sheu T.W.H., Finite element analysis of vortex shedding oscillations from cylinders in the straight channel, Computational Mechanics, 33(2), pp. 81-94, 2004.

[7] Prasanth, T. K., Behara, S., Singh S.P., Kumar, R., \& Mittal, S., "Effect of blockage on vortex-induced vibrations at low Reynolds numbers" Journal of Fluids and Structures, 22(6-7), pp. 865-876, 2006.

[8] Prasanth, T.K. \& Mittal, S., Vortex-induced vibrations of a circular cylinder at low Reynolds numbers, Journal of Fluid Mechanics, 594, pp. 463-491, 2008.

[9] Rodi, W., Comparison of LES and RANS calculations of the flow around bluff bodies, Journal of Wind Engineering and Industrial Aerodynamics, 69-71, pp. 55-75, 1997.

[10] Steggel, N., \& Rockliff, N., Simulations of the Effects of Body Shape on Lock-in Characteristics in Pulsating Flow by the Discrete Vortex Method, Journal of Wind Engineering and Industrial Aerodynamics, 69-71, pp. 317329, 1997.

[11] Larsen, A., \& Walther, J.H., Discrete Vortex Simulation of Flow around Five generic Bridge Deck Sections, Journal of Wind Engineering and Industrial Aerodynamics, 77-78, pp. 591-602, 1998.

[12] Lopes, A.V., Cunha, A., \& Simoes, L.M.C., Modelo Computacional de Análise Aeroelástica das Condiçoes de Utilizaçao de Estructuras Esbeltas, Congresso de Metodos Computacionais em Engenharia, Lisboa, Portugal, 2004.

[13] Vickery, B.J., \& Basu, R.I., Across-Wind Vibrations of Structures of Circular Cross-Section. Part I. Development of a Mathematical Model for Two-Dimensional Conditions, Journal Wind Engineering and Industrial Aerodynamics, 12, pp. 49-73, 1983.

[14] Fujarra, A.L.C., Pesce, C.P., Flemming, F., \& Williamson, C.H.K., Vortexinduced vibration of a flexible cantilever, Journal of Fluids and Structures, 15, pp. 651-658, 2001.

[15] Kitagawa, T., Fujino, Y., \& Kimura, K., Effects of free end condition on end-cell induced vibration, Journal of Fluids and Structures, 13, pp. 499$518,1999$.

[16] Foces, A., Garrido, J. A., Vasallo, A., One-Dimensional Model for the Analysis of Thin-Walled Composite Beams, Revista Internacional de Métodos Numéricos para Cálculo y Diseño en Ingeniería, 23, pp. 225-242, 2007.

[17] Rossi, R., Light Weight Structures: Structural Analysis and Coupling Issues, $\mathrm{Ph}$. D. dissertation, Università di Bologna, 2005.

[18] Codina, R., Pressure Stability in Fractional Step Finite Element Methods for Incompressible Flows, Journal of Computational Physics, 170(1), pp. 112-140, 2001.

[19] Codina, R., Stabilized finite element approximation of transient incompressible flows using orthogonal subscales, Computer Methods in Applied Mechanics and Engineering, 191(39-40), pp. 4295-4321, 2002. 